\title{
Some Calabi-Yau threefolds with obstructed deformations over the Witt vectors
}

\author{
Stefan Schröer
}

\begin{abstract}
Some smooth Calabi-Yau threefolds in characteristic two and three that do not lift to characteristic zero are constructed. These threefolds are pencils of supersingular K3-surfaces. The construction depends on Moret-Bailly's pencil of abelian surfaces and Katsura's analysis of generalized Kummer surfaces. The threefold in characteristic two turns out to be nonrigid.
\end{abstract}

\section{Introduction}

A fundamental result in deformation theory states that each infinitesimal deformation of a compact complex Kähler manifold $Y$ with trivial dualizing sheaf extends to a deformation of arbitrarily high order. In other words, the base of the versal deformation is smooth. This result is due to several authors (see Bogomolov [Bog78], Kawamata [Kaw92], Ran [Ran92], Tian [Tia87] and Todorov $[$ Tod80]).

It is open whether or to what extent this holds true over ground fields $k$ of characteristic $p>0$. A sufficient condition for unobstructedness involving crystalline cohomology groups and formal liftings to characteristic zero was given in [Sch03]. Deligne [Del81] proved that any K3-surface lifts projectively to characteristic zero. In contrast, Hirokado [Hir99] found a smooth threefold in characteristic $p=3$ that does not admit a formal lifting. Here the deformations over the Witt vectors $W=W(k)$ are necessarily obstructed.

A natural question to ask is what is the relation between obstructions for deformations over Artin $k$-algebras and deformations over Artin $W$-algebras? Unfortunately, there are very few examples that could shed some light on the situation. The goal of this paper is to construct some interesting new examples of Calabi-Yau threefolds $Y$ in characteristic $p=2$ and 3 that do not formally lift to characteristic zero.

Our examples are pencils of supersingular K3-surfaces over the projective line. The construction draws from the theory of supersingular abelian surfaces and generalized Kummer surfaces. The starting point is Moret-Bailly's nontrivial pencil of an abelian surface [Mor81]. It turns out that the present threefold $Y$ in characteristic $p=2$ has some nontrivial deformations over $k$-algebras. This seems to be the first example of nonliftable Calabi-Yau manifolds with nontrivial deformations. It would be interesting to know whether or not the Hodge-de Rham spectral sequence $H^{r}\left(Y, \Omega_{Y}^{s}\right) \Rightarrow \mathbb{H}^{r+s}\left(Y, \Omega_{Y}^{\bullet}\right)$ degenerates.

This paper is organized as follows. In $\S 1$ threefolds with trivial canonical class that are fibered in supersingular K3-surfaces are considered. At this stage we do not care about existence and merely deduce some properties from our assumptions. In $\S 2$ we see that it is impossible to lift such threefolds to characteristic zero, even in a formal way. We turn in $\S 3$ to the existence question.

Received 13 March 2003, accepted in final form 5 August 2003, published online 15 October 2004.

2000 Mathematics Subject Classification 14J28, 14J30, 14J32, $14 \mathrm{~K} 10$.

Keywords: Calabi-Yau manifold, supersingular K3-surface, generalized Kummer surface.

This journal is (C) Foundation Compositio Mathematica 2004. 


\section{S. SCHRÖER}

Here we use an approach of Moret-Bailly to construct threefolds with trivial canonical class that are fibered in supersingular abelian surfaces. The construction works precisely at the primes $p=2$ and 3 , but the two cases have different features. This becomes clear in $\S 4$ : here the associated family of Kummer surfaces easily gives examples, in characteristic $p=3$, of our nonliftable Calabi-Yau threefolds. This approach fails, however, in characteristic $p=2$ due to the results of Shioda and Katsura. We deal with this problem in $\S 5$ : discarding Kummer surfaces, we work with generalized Kummer surfaces by replacing the sign involution by an automorphism of order three. We compute the discriminant of the intersection form in $\S 6$, which relies on descent theory and theta groups. It turns out that discriminant jumps in pencils. This is crucial in $\S 7$ : here we construct the desired nonliftable Calabi-Yau threefold for $p=2$. The nontrivial deformations are detected by jumping discriminants. In $\S 8$ we compute the Artin-Mazur formal group $\Phi_{Y}^{3}$.

\section{Calculation of invariants}

Let $k$ be an algebraically closed ground field of characteristic $p>0$. Suppose that $Y$ is a smooth proper threefold with $\omega_{Y}=\mathcal{O}_{Y}$, endowed with a smooth morphism $f: Y \rightarrow \mathbb{P}^{1}$ such that all geometric fibers $Y_{\bar{t}}, t \in \mathbb{P}^{1}$, are K3-surfaces with Picard number $\rho\left(Y_{\bar{t}}\right)=22$. We shall see in the following sections that such threefold actually exists in characteristic $p=2$ and 3. In this section, we merely collect some consequences from the assumptions.

We start by computing the Hodge numbers $h^{0 i}(Y)=h^{i}\left(\mathcal{O}_{Y}\right)$.

Proposition 1.1. We have $h^{0}\left(\mathcal{O}_{Y}\right)=h^{3}\left(\mathcal{O}_{Y}\right)=1$ and $h^{1}\left(\mathcal{O}_{Y}\right)=h^{2}\left(\mathcal{O}_{Y}\right)=0$.

Proof. Clearly $h^{0}\left(\mathcal{O}_{Y}\right)=1$ because $Y$ is connected. The Leray-Serre spectral sequence for $f: Y \rightarrow$ $\mathbb{P}^{1}$ gives an exact sequence

$$
H^{1}\left(\mathbb{P}^{1}, f_{*} \mathcal{O}_{Y}\right) \longrightarrow H^{1}\left(Y, \mathcal{O}_{Y}\right) \longrightarrow H^{0}\left(\mathbb{P}^{1}, R^{1} f_{*} \mathcal{O}_{Y}\right) .
$$

The fibers $Y_{t}$ are connected, so $\mathcal{O}_{\mathbb{P}^{1}} \rightarrow f_{*} \mathcal{O}_{Y}$ is bijective, and the term on the left vanishes. The cohomology groups $H^{1}\left(Y_{t}, \mathcal{O}_{Y_{t}}\right)$ vanish for all $t \in \mathbb{P}^{1}$, hence $R^{1} f_{*} \mathcal{O}_{Y}=0$ by semicontinuity, so the term on the right vanishes as well. We conclude that $h^{1}\left(\mathcal{O}_{Y}\right)=0$. The remaining Hodge numbers follow from Serre duality.

Smooth proper $n$-folds $X$ with $\omega_{X}=\mathcal{O}_{X}$ and $h^{1}\left(\mathcal{O}_{X}\right)=\cdots=h^{n-1}\left(\mathcal{O}_{X}\right)=0$ are called CalabiYau $n$-folds. They play an important role in the classification of schemes with trivial canonical class [Bea83] and mirror symmetry. Our $Y$ is an example of a Calabi-Yau threefold.

Proposition 1.2. The algebraic fundamental group $\pi_{1}(Y, y)$ vanishes.

Proof. Let $y \in Y$ be a closed point and $z \in \mathbb{P}^{1}$ be its image. According to [Gro71a, Exposé X, Corollary 1.4], we have an exact sequence

$$
\pi_{1}\left(Y_{z}, y\right) \longrightarrow \pi_{1}(Y, y) \longrightarrow \pi_{1}\left(\mathbb{P}^{1}, z\right)
$$

The K3-surface $Y_{z}$ and the projective line $\mathbb{P}^{1}$ are simply connected, so the same holds for $Y$.

Let $\eta \in \mathbb{P}^{1}$ be the generic point. Then $Y_{\eta}$ is a K3-surface over the function field $\kappa(\eta)$. Choose an algebraic closure of $\kappa(\eta)$ and let $Y_{\bar{\eta}}$ be the corresponding geometric generic fiber.

Lemma 1.3. The Picard group $\operatorname{Pic}\left(Y_{\eta}\right)$ is free of $\operatorname{rank} \rho\left(Y_{\eta}\right)=22$, and the inclusion map $\operatorname{Pic}\left(Y_{\eta}\right) \rightarrow$ $\operatorname{Pic}\left(Y_{\bar{\eta}}\right)$ is bijective.

Proof. To simplify notation, set $S=Y_{\eta}$ and $K=\kappa(\eta)$, let $K \subset \tilde{K} \subset \bar{K}$ be separable and algebraic closures, and write $\tilde{S}=S \otimes \tilde{K}$ and $\bar{S}=S \otimes \bar{K}$. First note that the map $\operatorname{Pic}(\tilde{S}) \rightarrow \operatorname{Pic}(\bar{S})$ is bijective, because the Picard scheme $\mathrm{Pic}_{S / K}$ is smooth and zero dimensional. 


\section{CAlabi-Yau Threefolds}

According to Tsen's theorem, we have $\operatorname{Br}(K)=0$. This implies that the injection $\operatorname{Pic}(S) \subset$ $\operatorname{Pic}(S / K)$ is bijective, where the right-hand side denotes the group of rational points in $\operatorname{Pic}_{S / K}$. Note that $\operatorname{Pic}(S / K)=\operatorname{Pic}(\tilde{S})^{G}$, where $G=\operatorname{Gal}(\tilde{K} / K)$ is the Galois group. By assumption, $\operatorname{Pic}(\tilde{S})=\operatorname{Pic}(\bar{S})$ is free of rank 22 , so it remains to check that $G$ acts trivially on $\operatorname{Pic}(\tilde{S})$. The following argument suggested by the referee proves this.

Fix a prime power $l^{n}$ with $l \neq p$ and consider the constructible sheaf $\mu_{l^{n}}$ on $Y$, which is constant. Its direct image $R^{2} f_{*}\left(\mu_{l^{n}}\right)$ is a constructible sheaf, and locally constant because $f: Y \rightarrow \mathbb{P}^{1}$ is smooth [FK88, ch. I, Lemma 8.13]. Then it must be constant because $\mathbb{P}^{1}$ is simply connected. Passing to the generic fiber, we infer that the $G$-action on $H^{2}\left(\tilde{S}, \mu_{l^{n}}\right)$ is trivial. Using the Kummer sequence, we deduce that $G$ acts trivially on $\operatorname{Pic}(\tilde{S})$ as well.

Proposition 1.4. The Picard group $\operatorname{Pic}(Y)$ is a free group of rank $\rho(Y)=23$.

Proof. The closure of any Cartier divisor on $Y_{\eta}$ is a Cartier divisor on $Y$, because $Y$ is locally factorial. Therefore, the restriction map $\operatorname{Pic}(Y) \rightarrow \operatorname{Pic}\left(Y_{\eta}\right)$ is surjective. An invertible $\mathcal{O}_{Y}$-module that is trivial on $Y_{\eta}$ is trivial on some open neighborhood of $Y_{\eta}$. It is then trivial on the preimage of some nonempty open subset $U \subset \mathbb{P}^{1}$, because $f: Y \rightarrow \mathbb{P}^{1}$ is a closed map. Hence, it comes from a Cartier divisor supported by the preimage of $\mathbb{P}^{1}-U$. In other words, the sequence

$$
0 \longrightarrow \operatorname{Pic}\left(\mathbb{P}^{1}\right) \longrightarrow \operatorname{Pic}(Y) \longrightarrow \operatorname{Pic}\left(Y_{\eta}\right) \longrightarrow 0
$$

is exact. By Lemma 1.3, $\operatorname{Pic}\left(Y_{\eta}\right)$ is a free group of rank $\rho\left(Y_{\eta}\right)=22$, and the statement follows.

We now turn to the Brauer group $\operatorname{Br}(Y)=H^{2}\left(Y, \mathbb{G}_{m}\right)$, where the cohomology is taken with respect to the étale topology.

Proposition 1.5. The Brauer group $\operatorname{Br}(Y)$ is annihilated by $p$.

Proof. Set $S=Y_{\eta}$ and let $\bar{S}=Y_{\bar{\eta}}$, the geometric generic fiber. It follows from [Gro68, Corollary 1.8], that the restriction map $\operatorname{Br}(Y) \rightarrow \operatorname{Br}(S)$ is injective. The Leray-Serre spectral sequence for the structure morphism $g: S \rightarrow \operatorname{Spec} \kappa(\eta)$ yields a spectral sequence $H^{r}\left(\eta, R^{s} g_{*} \mathbb{G}_{m, S}\right) \Rightarrow H^{r+s}\left(S, \mathbb{G}_{m, S}\right)$. The stalk $\left(R^{1} g_{*} \mathbb{G}_{m, S}\right)_{\bar{\eta}}=\operatorname{Pic}(\bar{S})$ is a finitely generated free group of finite rank, so $H^{1}\left(\eta, R^{1} g_{*} \mathbb{G}_{m, S}\right)=0$. The group $H^{2}\left(\eta, g_{*} \mathbb{G}_{m, S}\right)=\operatorname{Br}(\eta)$ vanishes by Tsen's theorem. It follows that the edge map $\operatorname{Br}(S) \rightarrow H^{0}\left(\eta, R^{2} g_{*} \mathbb{G}_{m, S}\right)$ is injective. The latter group is contained in $\operatorname{Br}(\bar{S})$. Now $\bar{S}$ is a K3-surface with Picard number $\rho=22$ over an algebraically closed field. Artin's argument for [Art74, Theorem 1.7], shows that the abelian group $\operatorname{Br}(\bar{S})$ is annihilated by $p$.

Next we calculate the $l$-adic Betti numbers $b_{i}(Y)=\operatorname{dim}_{\mathbb{Q}_{l}} H^{i}\left(Y, \mathbb{Q}_{l}\right)$ and the Euler characteristic $e(Y)=\sum(-1)^{i} b_{i}(Y)$, where $l$ is any prime number different from $p$.

Proposition 1.6. The $l$-adic Betti numbers $b_{i}=b_{i}(Y)$ and Euler characteristic are as follows: $b_{0}=b_{6}=1, b_{1}=b_{3}=b_{5}=0, b_{2}=b_{4}=23$, and $e=48$.

Proof. Obviously $b_{0}=1$ because $Y$ is connected. Proposition 1.2 implies that $H^{1}\left(Y, \mathbb{Z} / l^{n} \mathbb{Z}\right)=0$ for each $n>0$, hence $b_{1}=0$. To proceed, consider the Leray-Serre spectral sequence

$$
H^{r}\left(\mathbb{P}^{1}, R^{s} f_{*}\left(\mu_{l^{n}}\right)\right) \Longrightarrow H^{r+s}\left(Y, \mu_{l^{n}}\right) .
$$

The constructible sheaves $R^{s} f_{*}\left(\mu_{l^{n}}\right)$ are constant, by the same argument as in the proof of Lemma 1.3. It follows from the proper base change theorem that $R^{s} f_{*}\left(\mu_{l^{n}}\right)=0$ for $s$ odd, because then the K3-surfaces $Y_{t}$ satisfy $H^{s}\left(Y_{t}, \mu_{l^{n}}\right)=0$. Therefore, the spectral sequence must degenerate. Moreover, $H^{r}\left(\mathbb{P}^{1}, R^{s} f_{*}\left(\mu_{l^{n}}\right)\right)$ vanishes for $r$ odd because $\mathbb{P}^{1}$ is simply connected.

From this we immediately deduce $H^{3}\left(Y, \mu_{l^{n}}\right)=0$ and hence $b_{3}=0$. The module $H^{2}\left(Y, \mu_{l^{n}}\right)$ is isomorphic to the sum of $H^{2}\left(\mathbb{P}^{1}, \mu_{l^{n}}\right)$, which has rank 1 , and $H^{0}\left(\mathbb{P}^{1}, R^{2} f_{*}\left(\mu_{l^{n}}\right)\right)$, which has rank 22 . 


\section{S. SCHRÖER}

The latter holds because by the stalks $R^{2} f_{*}\left(\mu_{l^{n}}\right)_{t}=H^{2}\left(Y_{t}, \mu_{l^{n}}\right)$ have rank 22 . Consequently $b_{2}=23$, and the remaining Betti numbers follow from Poincaré duality.

We finally observe that $Y$ carries an ample invertible sheaf.

Proposition 1.7. The scheme $Y$ is projective.

Proof. It suffices to check that the morphism $f: Y \rightarrow \mathbb{P}^{1}$ is projective. The generic fiber $Y_{\eta}$ is a regular surface, hence there is an ample invertible $\mathcal{O}_{Y_{\eta}}$-module $\mathcal{L}_{\eta}$. Extend it to an invertible $\mathcal{O}_{Y}$-module $\mathcal{L}$ and fix a point $t \in \mathbb{P}^{1}$. The specialization map $\operatorname{Pic}\left(Y_{\eta}\right) \rightarrow \operatorname{Pic}\left(Y_{t}\right)$ has finite cokernel, because all fibers have Picard number $\rho=22$. Hence, $\mathcal{L} \cdot C>0$ for any curve $C \subset Y_{t}$. We also have $\mathcal{L}_{t} \cdot \mathcal{L}_{t}=\mathcal{L}_{\eta} \cdot \mathcal{L}_{\eta}>0$. This implies that $\mathcal{L}_{t}=\left.\mathcal{L}\right|_{Y_{t}}$ is ample, and therefore $\mathcal{L}$ is $f$-ample.

\section{Nonliftability}

We keep the assumptions from the preceding section. We say that our Calabi-Yau threefold $Y$ admits a formal lifting to characteristic zero if there is a complete local noetherian ring $R$ with residue field $R / \mathfrak{m}=k$ containing $\mathbb{Z}$ as a subring, together with a proper flat formal $R$-scheme $\mathfrak{Z} \rightarrow \operatorname{Spf}(R)$ with closed fiber $\mathfrak{Z}_{0}=Y$. For example, a smooth proper scheme $X$ admits a formal lifting to characteristic zero if $H^{2}\left(X, \Theta_{X}\right)=0$. The first main result of this paper is as follows.

Theorem 2.1. The Calabi-Yau threefold $Y$ does not admit a formal lifting to characteristic zero.

Proof. Suppose to the contrary that there is a formal lifting $\mathfrak{Z} \rightarrow \operatorname{Spf}(R)$ to characteristic zero. We may assume that $R$ is integral, say with field of fractions $R \subset Q$. Choose a descending sequence of ideals $\mathfrak{m}=I_{0} \supset I_{1} \supset \cdots$ so that $I_{n} / I_{n+1}$ have length one. Let $Y_{n} \subset \mathfrak{Z}$ be the infinitesimal neighborhood of $Y$ defined by the ideal $I_{n+1} \mathcal{O}_{\mathfrak{Z}}$. The exact sequence

$$
H^{1}\left(Y, \mathcal{O}_{Y}\right) \rightarrow \operatorname{Pic}\left(Y_{n+1}\right) \longrightarrow \operatorname{Pic}\left(Y_{n}\right) \longrightarrow H^{2}\left(Y, \mathcal{O}_{Y}\right)
$$

implies that the restriction map $\operatorname{Pic}(\mathfrak{Z}) \rightarrow \operatorname{Pic}(Y)$ is bijective. According to Proposition 1.7, there is an ample invertible $\mathcal{O}_{Y}$-module, so Grothendieck's algebraization theorem tells us that $\mathfrak{Z}$ is algebraizable. Write $\mathfrak{Z}=Z^{\wedge}$ for some smooth proper $g: Z \rightarrow \operatorname{Spec}(R)$. Note that $\operatorname{Pic}(Z)=$ $\operatorname{Pic}(\mathfrak{Z})=\operatorname{Pic}(Y)$. In particular, the dualizing sheaf $\omega_{Z / R}$ is trivial, and the generic fiber $Z_{\eta}$ over $R$ is a Calabi-Yau threefold in characteristic zero.

Now Hirokado's argument [Hir99] yields a contradiction: using that the constructible sheaves $R^{3} g_{*}\left(\mu_{l^{n}}\right)$ are constant, we deduce $b_{3}\left(Z_{\bar{\eta}}\right)=b_{3}(Y)=0$. On the other hand, the Hodge-de Rham spectral sequence for $Z_{\bar{\eta}}$ degenerates, hence $b_{3}\left(Z_{\bar{\eta}}\right) \geqslant h^{3,0}\left(Z_{\bar{\eta}}\right)=1$, a contradiction.

As a consequence, the deformation functor $D_{Y}$ is not formally smooth. Here $D_{Y}$ is the functor which associates to an Artin algebra $R$ over the Witt vectors $W(k)$ the set $D_{Y}(R)$ of isomorphism classes of proper flat $R$-schemes $\mathfrak{Y}$ together with an identification $\mathfrak{Y}_{0} \simeq Y$.

Remark 2.2. The one-dimensional Calabi-Yau manifolds are elliptic curves, and Grothendieck showed that any smooth proper curve lifts to characteristic zero [Gro71a, Exposé III, Corollary 7.4]. The two-dimensional Calabi-Yau manifolds are the K3-surface. According to Deligne [Del81], every K3-surface lifts to characteristic zero. Hirokado's [Hir99] counterexample showed that one cannot hope for any general result such as this in higher dimensions. The upshot of this paper is that nonliftability might be quite frequent for Calabi-Yau manifolds.

\section{Moret-Bailly's pencil of abelian surfaces}

The task now is to construct pencils of K3-surfaces $Y$ as in $\S 1$. In this section we take a first step into that direction by constructing a pencil of abelian surfaces with similar properties. 


\section{Calabi-Yau threefolds}

Fix an algebraically closed field $k$ of characteristic $p>0$.

Let $A$ be the superspecial abelian surface, that is, $A$ is a two-dimensional abelian variety isomorphic to the product of two supersingular elliptic curves. Note that $A$ does not depend, up to isomorphism, on the choice of the supersingular elliptic curves by [Shi79, Theorem 3.5]. Its a-number

$$
a(A)=\operatorname{dim}_{k} \operatorname{Hom}_{\mathrm{gr}}\left(\alpha_{p}, A\right)
$$

equals $a(A)=2$, and we have an embedding of group schemes $\alpha_{p}^{\oplus 2} \subset A$. Actually, Oort proved in [Oor75, Theorem 2], that an abelian surface is superspecial if and only if its $a$-number is $a=2$.

Fix an integer $n \geqslant 1$ and an exact sequence

$$
0 \longrightarrow \mathcal{O}_{\mathbb{P}^{1}}(-n) \longrightarrow \mathcal{O}_{\mathbb{P}^{1}}^{\oplus 2} \longrightarrow \mathcal{O}_{\mathbb{P}^{1}}(n) \longrightarrow 0 .
$$

Such extensions correspond to pairs of sections $r, s \in H^{0}\left(\mathbb{P}^{1}, \mathcal{O}_{\mathbb{P}^{1}}(n)\right)$ without common zeros. Let $X^{\prime}=A \times \mathbb{P}^{1}$ be the constant relative abelian variety over $\mathbb{P}^{1}$, and let $H \subset X^{\prime}$ be the relative radical subgroup scheme of height $\operatorname{ht}(H)=1$ whose $p$-Lie algebra is isomorphic to

$$
\operatorname{Lie}\left(H / \mathbb{P}^{1}\right)=\mathcal{O}_{\mathbb{P}^{1}}(-n) \subset \mathcal{O}_{\mathbb{P}^{1}}^{\oplus 2}=\operatorname{Lie}\left(\alpha_{p}^{\oplus 2} / \mathbb{P}^{1}\right)=\operatorname{Lie}\left(X^{\prime} / \mathbb{P}^{1}\right),
$$

as explained in [Gro70, Exposé $\mathrm{VII}_{A}$, Theorem 7.2]. Here the inclusion $\mathcal{O}_{\mathbb{P}^{1}}(-n) \subset \mathcal{O}_{\mathbb{P}^{1}}^{\oplus 2}$ comes from the extension (1). This works because both the Lie bracket and $p$ th power operation are trivial for $\operatorname{Lie}\left(\alpha_{p}^{\oplus 2}\right)$.

The quotient $X=X^{\prime} / H$ is a relative abelian surface over $\mathbb{P}^{1}$, whose fibers $X_{t}, t \in \mathbb{P}^{1}$, are supersingular abelian surfaces. This construction is due to Moret-Bailly [Mor79], who considered the case $n=1$.

Proposition 3.1. We have $\operatorname{Lie}\left(X / \mathbb{P}^{1}\right) \simeq \mathcal{O}_{\mathbb{P}^{1}}(-n p) \oplus \mathcal{O}_{\mathbb{P}^{1}}(n)$.

Proof. Moret-Bailly showed this for $n=1$. In the general case, the family $X \rightarrow \mathbb{P}^{1}$ comes from the family with $n=1$ via pullback along the map $(r, s): \mathbb{P}^{1} \rightarrow \mathbb{P}^{1}$ of degree $n$. For the convenience of the reader we recall Moret-Bailly's arguments.

First note that $\operatorname{Lie}\left(X / \mathbb{P}^{1}\right)$ is dual to the conormal sheaf $N_{H / X^{\prime}}$ restricted to the zero section $\mathbb{P}^{1} \subset H$. To see this we use cotangent complexes (see [Gro71b, Exposé VIII] and [Ill71]). Consider the commutative diagram of cotangent complexes

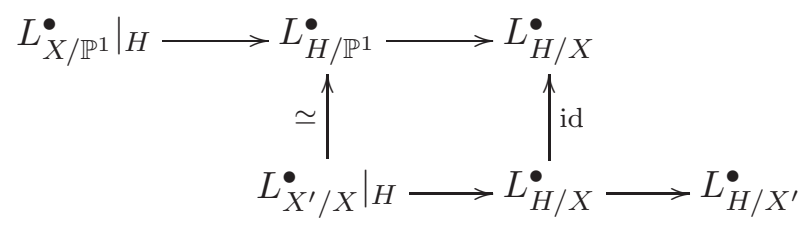

in the derived category of perfect complexes on $H$. Here the rows are distinguished triangles coming from the two compositions $H \rightarrow X \rightarrow \mathbb{P}^{1}$ and $H \rightarrow X^{\prime} \rightarrow X$. The vertical map on the left is the base change map induced from the following cartesian diagram.

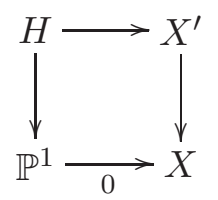

This base change map is a quasiisomorphism according to [Ill71, ch. II, Corollary 2.2.3], because $\mathcal{T}_{\text {or }}{ }_{r}^{\mathcal{O}_{X}}\left(\mathcal{O}_{X^{\prime}}, \mathcal{O}_{\mathbb{P}^{1}}\right)=0$ for $r>0$ since $X^{\prime} \rightarrow X$ is flat. It then follows that there is a quasiisomorphism $\left.L_{X / \mathbb{P}^{1}}\right|_{H} \rightarrow L_{H / X^{\prime}}^{\bullet}[-1]$ in the derived category, and the induced bijection on cohomology yields the desired identification $\left.\Omega_{X / \mathbb{P}^{1}}^{1}\right|_{H} \simeq N_{H / X^{\prime}}$. 


\section{S. SCHRÖER}

To calculate the latter, we may replace $X^{\prime}$ by $X^{\prime \prime}=\mathbb{G}_{a, \mathbb{P}^{1}}^{\oplus 2}$ via the canonical composition of closed embeddings

$$
\alpha_{p, \mathbb{P}^{1}}^{\oplus 2} \subset{ }_{p} X^{\prime} \subset \mathbb{G}_{a, \mathbb{P}^{1}}^{\oplus 2}
$$

as explained in [Mor79, p. 136]. Let $U, V \in H^{0}\left(\mathbb{P}^{1}, \mathcal{O}_{\mathbb{P}^{1}}(1)\right)$ be homogeneous coordinates, and write $\mathbb{G}_{a}^{\oplus 2}=\operatorname{Spec} k[x, y]$. Let $R=k[x, y, U, V]$ be the homogeneous coordinate ring for $X^{\prime \prime}$, and $I \subset R$ the homogeneous ideal for $H \subset X^{\prime \prime}$. Then $I=\left(y^{p}, x^{p}, s x-r y\right)$, where $r, s$ are the two homogeneous polynomials in $U, V$ of degree $n$ defining $\operatorname{Lie}(H) \subset \operatorname{Lie}\left(X^{\prime} / \mathbb{P}^{1}\right)=\operatorname{Lie}\left(X^{\prime \prime} / \mathbb{P}^{1}\right)$. The Hilbert-Burch theorem (see [Eis95, Theorem 20.12]) tells us that

$$
0 \longrightarrow R(-n) \oplus R(-n p) \stackrel{\varphi_{2}}{\longrightarrow} R \oplus R \oplus R(-n) \stackrel{\varphi_{1}}{\longrightarrow} I \longrightarrow 0
$$

is an exact sequence of graded $R$-modules where the maps are given by the matrices

$$
\varphi_{2}=\left(\begin{array}{cc}
0 & r^{p} \\
r y-s x & -s^{p} \\
x^{p} & (r y-s x)^{p-1}
\end{array}\right) \quad \text { and } \quad \varphi_{1}=\left(y^{p}, \quad x^{p}, \quad s x-r y\right) .
$$

Indeed, the entries of $-r^{p} \varphi_{1}$ are the $2 \times 2$-minors of $\varphi_{2}$ with the appropriate signs (note that the sequence in [Mor79, p. 137] corresponding to (2) is slightly incorrect). Tensoring the resolution with the residue ring $\bar{R}=R /(x, y)$, we obtain an exact sequence

$$
\bar{R}(-n) \oplus \bar{R}(-n p) \stackrel{\bar{\varphi}_{2}}{\longrightarrow} \bar{R} \oplus \bar{R} \oplus \bar{R}(-n) \stackrel{\bar{\varphi}_{1}}{\longrightarrow} I /(x, y) I \longrightarrow 0,
$$

which in turn gives an exact sequence

$$
0 \longrightarrow \bar{R}(-n p) \longrightarrow \bar{R} \oplus \bar{R} \oplus \bar{R}(-n) \stackrel{\bar{\varphi}_{1}}{\longrightarrow} I /(x, y) I \longrightarrow 0 .
$$

The map on the left is defined by the transpose of $\left(r^{p},-s^{p}, 0\right)$, and its cokernel is the graded $\bar{R}$-module $\bar{R}(n p) \oplus \bar{R}(-n)$. The letter defines the locally free $\mathcal{O}_{\mathbb{P}^{1}}$-module $\mathcal{O}_{\mathbb{P}^{1}}(n p) \oplus \mathcal{O}_{\mathbb{P}^{1}}(-n)$, which by construction is dual to $\operatorname{Lie}\left(X / \mathbb{P}^{1}\right)$.

This has an interesting consequence for the dualizing sheaf $\omega_{X}$.

Corollary 3.2. The dualizing sheaf $\omega_{X}$ is trivial if and only if either $p=3$ and $n=1$, or $p=2$ and $n=2$.

Proof. We have $\omega_{X / \mathbb{P}^{1}}=\mathcal{O}_{X}(n p-n)$ by Proposition 3.1 and $\omega_{\mathbb{P}^{1}}=\mathcal{O}_{\mathbb{P}^{1}}(-2)$, so $\omega_{X}=\mathcal{O}_{X}(n p-n-2)$. Consequently $\omega_{X}$ is trivial if and only if $n(p-1)=2$, and the result follows.

We shall see in the following sections how to obtain some interesting Calabi-Yau threefolds from $X$ as above.

\section{A pencil of Kummer surfaces}

We now examine the family $X \rightarrow \mathbb{P}^{1}$ of abelian surfaces constructed in the preceding section in the special case $p=3$ and $n=1$. This is the first of the two cases where $\omega_{X}$ is trivial.

Since $p \neq 2$ and $k$ is algebraically closed, the kernel ${ }_{2} X \subset X$ of the multiplication by two map [2] $: X \rightarrow X$ consists of sixteen disjoint sections. We may also view ${ }_{2} X$ as the relative fixed point scheme of the sign change map $[-1]: X \rightarrow X$. Let $Z=X /[-1]$ be the corresponding quotient scheme. It inherits a fibration $Z \rightarrow \mathbb{P}^{1}$ whose fibers $Z_{t}$ are singular Kummer surfaces. Let $D_{\eta} \subset Z_{\eta}$ be the reduced singular locus and $D \subset Z$ be its closure. By flatness, each fiber $D_{t}$ is the reduced singular locus. The 16 singular points on $Z_{t}$ are rational double points of type $A_{1}$. The blowing up of $D_{t}$ yields the minimal resolution. Let $X \rightarrow Z$ be the blowing up with center $D \subset Z$. Then $Y$ is a smooth threefold, and the fibers $Y_{t}$ of the induced family $Y \rightarrow \mathbb{P}^{1}$ are Kummer K3-surfaces. 


\section{CAlabi-Yau Threefolds}

Proposition 4.1. We have $\rho\left(Y_{\bar{t}}\right)=22$ for every $t \in \mathbb{P}^{1}$.

Proof. Since $E$ is supersingular, the Picard number of $A$ is $\rho(A)=6$, so $\rho\left(B_{\bar{t}}\right)=6$ as well. It then follows from [Shi79, Proposition 3.1], that $\rho\left(Y_{\bar{t}}\right)=16+6=22$.

Proposition 4.2. The dualizing sheaf $\omega_{Y}$ is trivial.

Proof. Let $\eta \in \mathbb{P}^{1}$ be the generic point. Then $\omega_{Y_{\eta}}$ is trivial, because $Y_{\eta}$ is a K3-surface. Hence, $\omega_{Y}=\mathcal{O}_{Y}(m)$ for some integer $m$. The relative dualizing sheaf $\omega_{Y / Z}$ is supported by the exceptional locus of $Y \rightarrow Z$. It must be trivial, because it is trivial on the generic fiber. We conclude that $\omega_{Z}=\mathcal{O}_{Z}(m)$. The canonical map $f: X \rightarrow Z$ is étale on $X-{ }_{2} X$, so the invertible sheaves $\omega_{X}$ and $f^{*}\left(\omega_{Z}\right)$ coincide outside ${ }_{2} X$. Since $X$ satisfies Serre's condition $\left(S_{2}\right)$ and ${ }_{2} X \subset X$ has codimension two, we must have $\omega_{X} \simeq f^{*}\left(\omega_{Z}\right)$ by [Har94, Theorem 1.12]. Consequently $m=0$, and $\omega_{Y}$ must be trivial.

We conclude that $Y$ satisfies the assumptions from $\S 1$, so $Y$ is a Calabi-Yau threefold with $b_{2}=23$ that does not admit a formal lifting to characteristic zero.

Note that the preceding approach does not work for $p=2$, because then $Z_{t}$ is a rational surfaces with an elliptic singularity (see [Kat87, Corollary 2.12], [Kat78, Theorem A], or [Shi74, Proposition 1]). We shall sidestep this problem in the following sections.

\section{Generalized Kummer surfaces}

In this section we construct some generalized Kummer surfaces. This terminology is due to Katsura [Kat87], and denotes K3-surfaces arising as minimal resolutions of quotients of abelian surfaces by finite group actions. Katsura mainly studied the case $p \geqslant 3$. Throughout we work in characteristic $p=2$. Let $B$ be an abelian surface. According to [Kat78, Theorem $\mathrm{A}]$, the corresponding singular Kummer surface $B /[-1]$ is birational to a K3-surface if and only if $B$ is ordinary, and a rational surface if and only if $B$ is supersingular. To obtain K3-surfaces from supersingular abelian surfaces, we replace the sign involution $[-1]$ by an automorphism of order three.

In this section our ground field $k$ contains $\mathbb{F}_{4}$, but is not necessarily algebraically closed. Let $E$ be the supersingular elliptic curve given by the Weierstrass equation $x^{3}=y^{2}+y$ and fix a primitive third root of unity $\zeta \in \mathbb{F}_{4}$. Let $\varphi: E \rightarrow E$ be the automorphism given by $(x, y) \mapsto(\zeta x, y)$. This defines an action of $G=\mathbb{Z} / 3 \mathbb{Z}$ on $E$. The induced representation on $H^{0}\left(E, \Omega_{E}^{1}\right)$ is scalar multiplication by $\zeta$, because $d x$ is an invariant differential form on $E$. The action on $E$ has three fixed points, namely $(0,0),(0,1)$, and $\infty$. Each fixed point $x \in E$ comes along with a representation $\rho_{x}: G \rightarrow \operatorname{Aut}_{\kappa(x)}\left(\mathfrak{m}_{x} / \mathfrak{m}_{x}^{2}\right)$ taking values in $\mathbb{F}_{4}^{\times} \subset \kappa(x)^{\times}$. The generator $\varphi$ maps to $\zeta \in \mathbb{F}_{4}^{\times}$under each $\rho_{x}$ because $\mathfrak{m}_{x} / \mathfrak{m}_{x}^{2}=\Omega_{E}^{1}(x)$.

Now consider the superspecial abelian surface $A=E \times E$, endowed with the action of $G$ via $\phi=(\varphi, \varphi)$. Let $\alpha_{2} \subset A$ be any embedding of group schemes.

Proposition 5.1. The subgroup scheme $\alpha_{2} \subset A$ is stable under the $G$-action.

Proof. We have to check that $\phi\left(\alpha_{2}\right)=\alpha_{2}$ as subschemes in $A$. The origin $x \in A$ is clearly a fixed point for $\phi$. Let $\mathfrak{m} \subset \mathcal{O}_{A, x}$ be the maximal ideal. The first-order infinitesimal neighborhood of $x$ is the spectrum of $R=k \oplus \mathfrak{m} / \mathfrak{m}^{2}$. As remarked above, $\phi$ acts via scalar multiplication by $\zeta$ on $\mathfrak{m} / \mathfrak{m}^{2}$. Hence, $G$ leaves any subscheme of $\operatorname{Spec}(R)$ invariant.

We see that the $G$-action on the superspecial abelian surface $A=E \times E$ descends to a $G$-action on the supersingular abelian surface $B=A / \alpha_{2}$. Its fixed points are easy to determine: we saw that $\varphi$ has precisely three fixed points. In turn, $\phi: A \rightarrow A$ has $9=3 \times 3$ fixed points, and the same holds on $B$. These fixed points correspond to the singularities on $B / G$, which is a proper normal surface. 


\section{S. SCHRÖER}

Proposition 5.2. The singularities on $B / G$ are rational double points of type $A_{2}$.

Proof. It suffices to check this for the origin $x \in A$, because we may replace any fixed point of $\varphi: E \rightarrow E$ by the origin of $E$. Let $\hat{E}$ be the formal completion at the origin $y \in E$, which is a formal group isomorphic to $\hat{\mathbb{G}}_{a}$ because $E$ is supersingular. Hence, there is a uniformizer $u \in \mathcal{O}_{E, y}^{\wedge}$ such that the multiplication $\hat{E} \times \hat{E} \rightarrow \hat{E}$ is given by the formal group law $u \mapsto u+u^{\prime}$. In turn, there is a regular system of parameters $u, v \in \mathcal{O}_{A, x}^{\wedge}$ such that $u \mapsto u+u^{\prime}$ and $v \mapsto v+v^{\prime}$ is the formal group law for $\hat{A}$.

Write $\alpha_{2}=\operatorname{Spec} k[\epsilon]$. The action $\alpha_{2} \times \hat{A} \rightarrow \hat{A}$ is given by $u \mapsto u+i \epsilon$ and $v \mapsto v+j \epsilon$ for some $i, j \in k$ that do not vanish simultaneously, say $j \neq 0$. A power series $f(u, v)$ is invariant under the $\alpha_{2}$-action if and only if $f(u, v)=f(u+i \epsilon, v+j \epsilon)$. For example, $x^{\prime}=u^{2}$ and $y^{\prime}=u+v i / j$ are $\alpha_{2}$-invariants. Using that $\mathcal{O}_{A, x}^{\wedge}$ is a free module of rank two over $\mathcal{O}_{B, x}^{\wedge}$ by [Ser65, p. IV-37, Proposition 22], we infer that $x^{\prime}, y^{\prime} \in \mathcal{O}_{B, x}^{\wedge}$ is a regular system of parameters. The induced $G$-action has $\phi^{*}\left(x^{\prime}\right) \equiv \zeta^{2} x^{\prime}$ and $\phi^{*}\left(y^{\prime}\right) \equiv \zeta y^{\prime}$ modulo $\mathfrak{m}^{2}$, where $\mathfrak{m} \subset \mathcal{O}_{B, x}^{\wedge}$ denotes the maximal ideal. Using Lemma 5.4 below, we find another regular system of parameters $x, y \in \mathcal{O}_{B, x}^{\wedge}$ with $\phi^{*}(x)=\zeta^{2} x$ and $\phi^{*}(y)=\zeta y$. The $G$-invariants are then generated by $x^{3}, x y, y^{3}$, and this means that $B / G$ acquires a rational double point of type $A_{2}$.

A local computation shows that blowing up the reduced singular locus in a rational double point of type $A_{2}$ yields the minimal resolution, and the exceptional divisor consists of two smooth rational $(-2)$-curves intersecting transversely.

Theorem 5.3. The minimal resolution $S$ of $B / G$ is a K3-surface.

Proof. The $G$-action on $B$ has the following four properties. First, it has no fixed curves. Second, the generator of $G$ has some isolated fixed points. Third, all singularities on $B / G$ are rational singularities by Proposition 5.2. Finally, the induced $G$-action on $H^{0}\left(B, \omega_{B}\right)=k$ is trivial. To see this choose a fixed point $x \in B$. Let $\mathfrak{m} \subset \mathcal{O}_{B, x}$ be the maximal ideal. We saw that the $k$-linear $G$-action on $\mathfrak{m} / \mathfrak{m}^{2}$ is diagonizable with eigenvalues $\zeta, \zeta^{2}$. Using $\operatorname{det}\left(\mathfrak{m} / \mathfrak{m}^{2}\right)=\Omega_{B}^{2}(x)$, we infer that $G$ must act via $1=\zeta \cdot \zeta^{2}$ on global 2 -forms.

Assuming $p \neq 2$, Katsura showed that under the preceding four conditions the minimal resolution $S$ of $B / G$ is a K3-surface [Kat87, Theorem 2.4 and Lemma 2.7]. Actually, he showed without any assumption on the characteristic that $\omega_{S}$ is trivial, and that $S$ is neither abelian, hyperelliptic, nor quasihyperelliptic. It then follows from the classification of surfaces that $S$ is either a K3-surface or a nonclassical Enriques surface [BM77]. The latter exists only in characteristic $p=2$. An Enriques surface, however, has by definition $l$-adic Betti number $b_{2}=10$. On the other hand we have $b_{2}(S)>18$, due to the exceptional curves coming from the nine rational double points of type $A_{2}$ on $B / G$. Therefore, $S$ must be a K3-surface.

We have used the following fact in the proof of Proposition 5.2. We include a proof due to the lack of a reference.

Lemma 5.4. Let $R$ be a regular local noetherian $k$-algebra and $f: R \rightarrow R$ an $k$-automorphism of finite order $m>0$ prime to the characteristic $p$. If $k$ contains a primitive $m$ th root of unity $\zeta$, then there is a regular system of parameters $u_{1}, \ldots, u_{d} \in R^{\wedge}$ and integers $n_{1}, \ldots, n_{d}$ such that $f\left(u_{i}\right)=\zeta^{n_{i}} u_{i}$.

Proof. Let $\mathfrak{m} \subset R$ be the maximal ideal. The induced $k$-linear map on $\mathfrak{m} / \mathfrak{m}^{2}$ is diagonizable with eigenvalues of the form $\zeta^{n_{i}}$, because $m$ is prime to $p$ and $\zeta \in k$. Hence, there is a regular system of parameters $u_{i} \in R$ whose classes modulo $\mathfrak{m}^{2}$ are eigenvectors for the eigenvalues $\zeta^{n_{i}}$. Suppose we have a homogeneous ideal $I \subset R$ with $f\left(u_{i}\right) \equiv \zeta^{n_{i}} u_{i}$ modulo $I$. If $I \neq 0$, then choose a 


\section{CAlabi-YAu ThreefoldS}

homogeneous subideal $J \subsetneq I$ so that $I=J+k u^{l}$, with the usual multiindex notation $u^{l}=u_{1}^{l_{1}} \ldots u_{d}^{l_{d}}$. Then $f\left(u_{i}\right) \equiv \zeta^{n_{i}}+y u^{l}$ modulo $J$ for some $y \in k$. We distinguish two cases. If $m$ divides $e=\sum l_{i} n_{i}$, then the congruence

$$
u_{i}=f^{m}\left(u_{i}\right) \equiv \zeta^{m n_{i}} u_{i}+y u^{l}\left(1+\zeta^{e}+\cdots+\zeta^{e(m-1)}\right)=u_{i}+y u^{l} m
$$

modulo $J$ implies $y=0$. If $m$ does not divide $e$, then the congruence

$$
f\left(u_{i}+x u^{l}\right) \equiv \zeta^{n_{i}} u_{i}+y u^{l}+x \zeta^{e} u^{l}=\zeta^{n_{i}}\left(u_{i}+x u^{l}\right)+u^{l}\left(y+x\left(\zeta^{e}-1\right)\right)
$$

modulo $J$ shows that we may choose $x \in k$ so that $y+x\left(\zeta^{e}-1\right)=0$ and then replace $u_{i}$ by $u_{i}+x u^{l}$. The principle of noetherian induction finishes the proof.

\section{Discriminants and Artin invariants}

We keep the notation as in the preceding section, such that $A=E \times E$ and $B=A / \alpha_{2}$ for some embedding $\alpha_{2} \subset A$. We saw that the minimal resolution $S$ of $B / G$ is a K3-surface, so $\operatorname{Pic}(S)$ is a free group of rank $\rho(S) \leqslant 22$. The goal of this section is to calculate the discriminant of this intersection pairing on $\operatorname{Pic}(S)$. It turns out that it depends in a subtle way on the position of $\alpha_{2} \subset A$, and this will be crucial in $\S 7$.

First, we examine the effect of the isogeny $g: A \rightarrow B$ on Picard groups. Consider the following four elliptic curves $C_{i} \subset A$. Set $C_{1}=E \times 0$ and $C_{2}=0 \times E$, let $C_{3}=\Delta_{E}$ be the diagonal subscheme, and let $C_{4}=\Gamma_{\varphi}$ be the graph of the automorphisms $\varphi: E \rightarrow E$. Note that $\Delta_{E} \cap \Gamma_{\varphi}$ is the fixed scheme of $\varphi: E \rightarrow E$, which consists of three reduced points, hence $C_{3} \cdot C_{4}=3$.

Let $V_{A}$ be the free abelian group generated by the $C_{i}$. To simplify calculations, we introduce another basis $C_{i}^{\prime}$ for $V_{A}$ as follows: set $C_{i}^{\prime}=C_{i}$ for $i=1,2$, and $C_{i}^{\prime}=C_{i}-C_{1}-C_{2}$ for $i=3,4$. The corresponding intersection matrix has an orthogonal decomposition

$$
\left(C_{i}^{\prime} \cdot C_{j}^{\prime}\right)=\left(\begin{array}{ll}
0 & 1 \\
1 & 0
\end{array}\right) \oplus\left(\begin{array}{cc}
-2 & 1 \\
1 & -2
\end{array}\right)
$$

hence $\operatorname{disc}\left(V_{A}\right)=-3$. It follows that the map $V_{A} \rightarrow \mathrm{NS}(A)$ is injective, and we may regard $V_{A}$ as a subgroup of either $\operatorname{Pic}(A)$ or $\operatorname{NS}(A)$.

The exact sequence $0 \rightarrow \alpha_{2} \rightarrow A \rightarrow B \rightarrow 0$ induces an exact sequence

$$
0 \rightarrow \operatorname{Hom}\left(\alpha_{2}, \mathbb{G}_{m}\right) \rightarrow \mathrm{Pic}_{B / k}^{0} \rightarrow \mathrm{Pic}_{A / k}^{0} \rightarrow 0
$$

by [Mum70, Theorem 1, p. 143]. So $\operatorname{Pic}(B) \rightarrow \operatorname{Pic}(A)$ is injective, and we can form the intersection $V_{B}=V_{A} \cap \operatorname{Pic}(B)$ inside $\operatorname{Pic}(A)$. Note that we may regard $V_{B}$ as either a subgroup $V_{B} \subset \operatorname{Pic}(B)$ or as a subgroup $V_{B} \subset \operatorname{Pic}(A)$.

Proposition 6.1. The group $V_{A} / V_{B}$ is annihilated by 2 .

Proof. Given an invertible $\mathcal{O}_{A}$-module $\mathcal{L}$, let $K(\mathcal{L}) \subset A$ be the kernel of the morphism $A \rightarrow \operatorname{Pic}_{A / k}^{0}$, $x \mapsto T_{x}^{*}(\mathcal{L}) \otimes \mathcal{L}^{\vee}$. According to [Mor81, Corollary 4.1.1 and Theorem 2.3], we have $\mathcal{L}=g^{*}(\mathcal{N})$ for some invertible $\mathcal{O}_{B}$-module $\mathcal{N}$ if and only if $\alpha_{2} \subset K(\mathcal{L})$. For $\mathcal{L}_{i}=\mathcal{O}_{A}\left(C_{i}\right)$ we have $K\left(\mathcal{L}_{i}\right)=C_{i}$ and $K\left(\mathcal{L}_{i}^{\otimes 2}\right)=2 C_{i}$, by considering the quotient morphism $A \rightarrow A / C_{i}$. Obviously we have $\alpha_{2} \subset 2 C_{i}$, so $\mathcal{L}_{i}^{\otimes 2}$ lies in $V_{B}$.

The group $V_{B}$ is closely related to $\operatorname{Pic}(S)$ and $\operatorname{Pic}(B / G)$.

Proposition 6.2. The map $\operatorname{Pic}(B / G) \rightarrow \mathrm{NS}(A)$ factors through $V_{B} \subset \mathrm{NS}(A)$.

Proof. We have $\rho(B / G) \leqslant 4$ because $\rho(S) \leqslant 22$ and there are 18 exceptional curves for the resolution of singularities $S \rightarrow B / G$. The elliptic curves $C_{i} \subset A$ are $G$-invariant, and $2 C_{i}$ are $\alpha_{2}$-invariant. 


\section{S. SCHRÖER}

Let $h: A \rightarrow B / G$ be the canonical morphism and $D_{i}=h\left(C_{i}\right)$. The $D_{i} \subset B / G$ are integral Weil divisors. Since the singularities on $B / G$ are rational double points of type $A_{2}$, the Weil divisors $3 D_{i}$ are Cartier. By construction, $h^{*}\left(3 D_{i}\right)=n_{i} C_{i}$ for some integers $n_{i} \geqslant 1$. It follows that $\operatorname{Pic}(B / G) \rightarrow$ $\mathrm{NS}(A)$ factors over $\mathrm{NS}(A) \cap\left(V_{A} \otimes \mathbb{Q}\right)$. Since $\operatorname{disc}\left(V_{A}\right)=-3$ is square free, the subgroup $V_{A} \subset \operatorname{NS}(A)$ is a direct summand, so the map factors over $V_{A}$, and therefore over $V_{B}$ as well.

The subgroup $V_{B} \subset V_{A}$ depends on the position of $\alpha_{2} \subset A$. Let $x \in A$ be the origin, and choose a regular system of parameters $u, v \in \mathcal{O}_{A, x}^{\wedge}$ such that $u \mapsto u+u^{\prime}, v \mapsto v+v^{\prime}$ is the formal group law for the formal group $A^{\wedge}$. Write $\alpha_{2}=\operatorname{Spec} k[\epsilon]$. Then the embedding $\alpha_{2} \rightarrow A^{\wedge}$ is of the form $u \mapsto u+i \epsilon$ and $v \mapsto v+j \epsilon$ for some $(i, j) \in \mathbb{A}^{2}(k)-\{0\}$. The subgroup scheme $\alpha_{2} \subset A$ depends only on the homogeneous coordinates $(i: j) \in \mathbb{P}^{1}(k)$. Let us regard the $\mathbb{F}_{4}$-valued points $\mathbb{P}^{1}\left(\mathbb{F}_{4}\right)$ as a subset of the $k$-valued points $\mathbb{P}^{1}(k)$.

Proposition 6.3. Set $v=\operatorname{dim}_{\mathbb{F}_{2}}\left(V_{A} / V_{B}\right)$. Then $v=3$ if the homogeneous coordinates satisfy $(i: j) \in \mathbb{P}^{1}\left(\mathbb{F}_{4}\right)$, and $v=4$ otherwise.

Proof. Consider an invertible $\mathcal{O}_{A}$-module of the form $\mathcal{L}=\mathcal{O}_{A}\left(\sum_{i=1}^{4} n_{i} C_{i}^{\prime}\right)$, where $n_{i} \in\{0,1\}$ and at least one coefficient $n_{i}$ is nonzero. The idea is to check whether $\alpha_{2} \subset A$ might be contained in $K(\mathcal{L}) \subset A$. First note that $K(\mathcal{L})$ is a finite group scheme of length $\chi(\mathcal{L})^{2}$ if $\chi(\mathcal{L}) \neq 0$, and of dimension $>0$ otherwise, according to [Mum70, p. 150]. The Riemann-Roch theorem and $n_{i}^{2}=n_{i}$ gives

$$
\chi(\mathcal{L})=\left(\sum_{i=1}^{4} n_{i} C_{i}^{\prime}\right)^{2} / 2=n_{1} n_{2}-n_{3}-n_{4}+n_{3} n_{4} .
$$

If $\chi(L)$ is odd, then $K(\mathcal{L})$ is an finite group scheme of odd length, hence $\alpha_{2} \not \subset K(\mathcal{L})$. The number $\chi(\mathcal{L})$ is odd except if $\left(n_{1}, \ldots, n_{4}\right)$ is of the form

$$
(1,0,0,0), \quad(0,1,0,0), \quad(1,1,1,0), \quad(1,1,0,1), \quad(1,1,1,1) .
$$

In these cases we compute the group schemes $K(\mathcal{L}) \subset A$. In the first four cases we have $\sum_{i=1}^{4} n_{i} C_{i}^{\prime}=$ $C_{j}$ for some $1 \leqslant j \leqslant 4$, and hence $K(\mathcal{L})=C_{j}$.

It remains to treat the last case $\sum_{i=1}^{4} C_{i}^{\prime}$. Modulo $2 V_{A} \subset \operatorname{Pic}(A)$, this divisor is linearly equivalent to

$$
C=C_{1}^{\prime}+C_{2}^{\prime}-3 C_{3}^{\prime}-3 C_{4}^{\prime}
$$

so $\sum_{i=1}^{4} C_{i}^{\prime} \in V_{B}$ if and only if $C \in V_{B}$. Consider the graph $C_{5}=\Gamma_{\varphi^{2}}$ of the automorphism $\varphi^{2}=$ $\varphi^{-1}: E \rightarrow E$. Since $C_{5}$ is $G$-invariant and $2 C_{5}$ is $\alpha_{2}$-invariant, some multiple of $\mathcal{O}_{A}\left(C_{5}\right) \in \operatorname{Pic}(A)$ is the preimage of some class in $\operatorname{Pic}(B / G)$. By Proposition 6.2, we must have $C_{5} \in V_{A} \subset \operatorname{NS}(A)$. However, then $C$ must be numerically equivalent to $C_{5}$, because $C \cdot C_{i}=C_{5} \cdot C_{i}$ for $1 \leqslant i \leqslant 4$. In turn, we have $K\left(\mathcal{O}_{A}(C)\right)=K\left(\mathcal{O}_{A}\left(C_{5}\right)\right)=C_{5}$.

Now we are done: recall that $\varphi: E \rightarrow E$ was defined on affine coordinates by $(x, y) \mapsto(\zeta x, y)$. An easy calculation shows that $(i: j) \in \mathbb{P}^{1}\left(\mathbb{F}_{4}\right)$ holds if and only if $\alpha_{2} \subset A$ is contained in precisely one $C_{j}$ from the elliptic curves $C_{1}=E \times 0, C_{2}=0 \times E, C_{3}=\Delta_{E}, C_{4}=\Gamma_{\varphi}$, and $C_{5}=\Gamma_{\varphi^{2}}$. In this situation we have $V_{B}=2 V_{A}+\mathbb{Z} C_{j}$ and $v=3$. If $(i: j) \notin \mathbb{P}^{1}\left(\mathbb{F}_{4}\right)$, then $V_{B}=2 V_{A}$ and $v=4$.

We now come to the main result of this section.

TheOREM 6.4. The group $\operatorname{Pic}(S)$ of the K3-surface $S$ is free of rank $\rho(S)=22$. The discriminant $d \in \mathbb{Z}$ of the intersection pairing on $\operatorname{Pic}(S)$ is $d=-2^{2}$ if the homogeneous coordinates satisfy $(i: j) \in \mathbb{P}^{1}\left(\mathbb{F}_{4}\right)$, and $d=-2^{4}$ otherwise.

Proof. We have $d=-2^{n}$ for some integer $n \geqslant 0$ as explained in [Art74, p. 556]. To determine this integer it suffices to compute the discriminant of the 2 -adic intersection form $\operatorname{Pic}(S) \otimes \mathbb{Z}_{2}$ up to units. 


\section{CAlabi-Yau threefolds}

The intersection matrix with respect to the exceptional divisors for the resolution of singularities $S \rightarrow B / G$ is

$$
\bigoplus_{i=1}^{9}\left(\begin{array}{cc}
-2 & 1 \\
1 & -2
\end{array}\right)
$$

Its discriminant is the unit $27 \in \mathbb{Z}_{2}$, hence $\operatorname{Pic}(B / G) \otimes \mathbb{Z}_{2} \subset \operatorname{Pic}(S) \otimes \mathbb{Z}_{2}$ is an orthogonal direct summand, and it suffices to compute the discriminant of $\operatorname{Pic}(B / G) \otimes \mathbb{Z}_{2}$.

We claim that the inclusion $\operatorname{Pic}(B / G) \otimes \mathbb{Z}_{2} \subset V_{B} \otimes \mathbb{Z}_{2}$ from Proposition 6.2 is bijective. Indeed, if $g: B \rightarrow B / G$ denotes the projection map, we have $g_{*} g^{*}(D)=3 D$ for any divisor $D \subset B / G$ by the projection formula. This implies that the inclusion $\operatorname{Pic}(B / G) \subset V_{B}$, which has finite index, becomes bijective after tensoring with $\mathbb{Z}_{2}$. Moreover, the discriminants of the intersection forms on $\operatorname{Pic}(B / G)$ and $V_{B} \subset \operatorname{Pic}(B)$ differ only by a power of the 2 -adic unit $3 \in \mathbb{Z}_{2}$.

For our purpose it suffices to compute the discriminant of $V_{B}$ endowed with the intersection form from $V_{B} \subset \operatorname{Pic}(B)$. Let us write $V_{B}^{\prime}$ for the same module $V_{B}$, but endowed with the intersection form from $V_{B} \subset \operatorname{Pic}(A)$. Then $\operatorname{disc}\left(V_{B}\right)=2^{-4} \operatorname{disc}\left(V_{B}^{\prime}\right)$. Indeed, if $h: A \rightarrow B$ denotes the projection map, then $2(\mathcal{L} \cdot \mathcal{L})=\left(\mathcal{L}_{A} \cdot \mathcal{L}_{A}\right)$ for any $\mathcal{L} \in \operatorname{Pic}(B)$.

This does it: we have $\operatorname{disc}\left(V_{A}\right)=-3$ and $V_{A} / V_{B} \simeq\left(V_{A} \otimes \mathbb{Z}_{2}\right) /\left(V_{B} \otimes \mathbb{Z}_{2}\right)$. According to [Ser79, Corollary, p. 49], this gives

$$
\operatorname{disc}\left(V_{B}^{\prime} \otimes \mathbb{Z}_{2}\right)=\operatorname{disc}\left(V_{A} \otimes \mathbb{Z}_{2}\right) \cdot \operatorname{ord}\left(V_{A} / V_{B}\right)^{2}=-3 \cdot 2^{2 v},
$$

where $v=\operatorname{dim}_{\mathbb{F}_{2}}\left(V_{A} / V_{B}\right)$. Using Proposition 6.3 , we infer that up to 2-adic units $\operatorname{Pic}(B / G)$ has discriminant $2^{2 \cdot 3-4}=2^{2}$ if $(i: j) \in \mathbb{P}^{1}\left(\mathbb{F}_{4}\right)$, and $2^{2 \cdot 4-4}=2^{4}$ otherwise.

For a K3-surface in characteristic $p>0$ with Picard number $\rho=22$ and discriminant $d=-p^{2 \sigma_{0}}$, the integer $\sigma_{0}$ is called the Artin invariant. We see that our K3-surface $S$ has Artin invariant $\sigma_{0}(S)=1$ or $\sigma_{0}(S)=2$.

\section{Nonrigidity}

Let $k$ be an algebraically closed field of characteristic $p=2$. Up to isomorphism, there is only one supersingular elliptic curve $E$, which is given by the Weierstrass equation $x^{3}=y^{2}+y$. Set $A=E \times E$ and $X^{\prime}=A \times \mathbb{P}^{1}$, and choose an exact sequence

$$
0 \longrightarrow \mathcal{O}_{\mathbb{P}^{1}}(-2) \stackrel{r, s}{\longrightarrow} \mathcal{O}_{\mathbb{P}^{1}}^{\oplus 2} \longrightarrow \mathcal{O}_{\mathbb{P}^{1}}(2) \longrightarrow 0
$$

given by two homogeneous quadratic polynomials $r, s \in H^{0}\left(\mathbb{P}^{1}, \mathcal{O}_{\mathbb{P}^{1}}(2)\right)$ without common zeros. As explained in $\S 3$, this defines a relative subgroup scheme $H \subset X^{\prime}$, whose quotient $X=X^{\prime} / H$ has $\omega_{X}=\mathcal{O}_{X}$. As in the preceding section, the group $G=\mathbb{Z} / 3 \mathbb{Z}$ acts on $E$, hence diagonally on $A$, and the induced fiberwise action on $X^{\prime} \rightarrow \mathbb{P}^{1}$ descends to a fiberwise action on $X \rightarrow \mathbb{P}^{1}$. Set $Z=X / G$ and let $Y \rightarrow Z$ be the minimal resolution of singularities. The same arguments as in $\S 4$ show that $\omega_{Y}=\mathcal{O}_{Y}$, and that the fibers $Y_{t}$ for the induced fibration $f: Y \rightarrow \mathbb{P}^{1}$ are K3-surfaces with Picard number $\rho\left(Y_{t}\right)=22$. So the smooth threefold $Y$ satisfies the assumptions of $\S 1$, hence is a Calabi-Yau threefold in characteristic $p=2$ without any formal lifting to characteristic zero. In particular, the deformations over the $W(k)$-algebras are obstructed. However, we shall see that there are many unobstructed deformations over $k$-algebras.

Proposition 7.1. The fiber $Y_{t}$ has Artin invariant $\sigma_{0}\left(Y_{t}\right)=1$ if and only if the homogeneous coordinates satisfy $(r(t): s(t)) \in \mathbb{P}^{1}\left(\mathbb{F}_{4}\right)$, and $\sigma_{0}\left(Y_{t}\right)=2$ otherwise.

Proof. This follows from Proposition 6.4 and the definition of $Y_{y}$ as the minimal resolution of the quotient of $X_{t}=X_{t}^{\prime} / H_{t}$ by $G$. 


\section{S. SCHRÖER}

Choose homogeneous coordinates $t=\left(t_{0}: t_{1}\right)$ for $\mathbb{P}^{1}$ and consider, for example, the case $r=t_{0}^{2}$ and $s=t_{1}^{2}$. Then $(r: s)=\left(t_{0}^{2}: t_{1}^{2}\right)$ is $\mathbb{F}_{4}$-valued if and only if $\left(t_{0}: t_{1}\right)$ is $\mathbb{F}_{4}$-valued, and there are precisely five such points on $\mathbb{P}^{1}$.

Now consider the case that $r=t_{0}^{2}+a t_{0} t_{1}$ and $s=t_{1}^{2}$, where $a \in k$ is a nonzero element. The quotient $r / s=\left(t_{0} / t_{1}\right)^{2}+a\left(t_{0} / t_{1}\right)=P\left(t_{0} / t_{1}\right)$ is given by the separable polynomial $P(x)=x^{2}+a x$, hence $P(x)=b$ has two distinct roots for each $b \in \mathbb{F}_{4}$. This means that there are precisely 8 points on $\mathbb{P}^{1}$ with $t_{1} \neq 0$ for which $(r(t): s(t))$ is $\mathbb{F}_{4}$-valued, plus the additional point (1:0).

This observation leads to nontrivial deformations over the formal power series $\operatorname{ring} R=k[[T]]$ : consider the two quadrics $r=t_{0}^{2}+T t_{0} t_{1}$ and $s=t_{1}^{2}$ with coefficients from $R$. It is easy to see that the construction of our Calabi-Yau threefolds carries over to a relative situation, so the two quadrics $r, s$ define a family $Y \rightarrow \operatorname{Spec}(R)$ of threefolds. The second main result of this paper is that this are nontrivial deformations as follows.

THEOREM 7.2. The deformation $Y \rightarrow \operatorname{Spec}(R)$ is not formally isomorphic to the trivial deformation $Y_{0} \otimes R \rightarrow \operatorname{Spec}(R)$.

Proof. Suppose to the contrary that these deformations are formally isomorphic. Then they are isomorphic and, in particular, their generic geometric fibers $Y_{L}$ and $Y_{0} \otimes L$ are isomorphic, where $L$ is the algebraic closure of $k((T))$. However, we saw above that the $Y_{0} \otimes L$ has five fibers over $\mathbb{P}_{L}^{1}$ whose Artin invariant is $\sigma_{0}=1$, whereas $Y_{L}$ has nine such fibers, a contradiction.

\section{The Artin-Mazur formal group}

We now go back to the situation in $\S 1$, such that $Y$ is a smooth proper threefold with $\omega_{Y}=\mathcal{O}_{Y}$ endowed with a smooth morphism $f: Y \rightarrow \mathbb{P}^{1}$ whose geometric fibers are K3-surfaces with $\rho=22$ in characteristic $p>0$. The goal of this section is to compute the Artin-Mazur formal group $\Phi_{Y}^{3}$ of such Calabi-Yau threefolds. Let me recall some definitions: if $A$ is a local Artin $k$-algebra with maximal ideal $I \subset A$, we may view the sheaf $1+I \otimes_{k} \mathcal{O}_{Y}$ as an abelian subsheaf of $\left(A \otimes_{k} \mathcal{O}_{Y}\right)^{\times}$. This gives a functor of Artin rings $\Phi_{Y}^{3}(A)=H^{3}\left(Y, 1+I \otimes_{k} \mathcal{O}_{Y}\right)$. Artin and Mazur proved in [AM77, Proposition 1.8], that, for schemes like $Y$, this functor is representable by a one-dimensional formal group.

Proposition 8.1. The Artin-Mazur formal group $\Phi_{Y}^{3}$ of our Calabi-Yau threefold $Y$ is isomorphic to the formal additive group $\hat{\mathbb{G}}_{a}$.

Proof. The referee suggested the following direct argument. Let $W=W(k)$ be the ring of Witt vectors, and $W \subset K$ be its quotient field. It follows from [KM74, Corollary 1], that the $l$-adic Betti numbers $b_{i}$ for the projective smooth scheme $Y$ coincide with the $W$-rank of the crystalline cohomology groups $H^{i}\left(Y / W, \mathcal{O}_{Y / W}\right)$. In particular, $H^{3}\left(Y / W, \mathcal{O}_{Y / W}\right) \otimes K=0$. On the other hand, the part of the $F$-crystal $H^{3}\left(Y / W, \mathcal{O}_{Y / W}\right) \otimes K$ whose slopes fall into the interval $[0,1)$ is the Dieudonné module for the maximal $p$-divisible quotient of the formal group $\Phi_{Y}^{3}$ (compare [Ill79, Remark 5.13] and [AM77, Corollary 3.3]). It then follows from the classification of one-dimensional formal groups that $\Phi_{Y}^{3}=\hat{\mathbb{G}}_{a}$.

It follows from a result of Van der Geer and Katsura [VK00, Proposition 16.4], that the Frobenius operator $F$ on the Witt vector cohomology $H^{3}\left(Y, W_{i} \mathcal{O}_{Y}\right)$ vanishes for all $i \geqslant 1$. Indeed, the Witt vector cohomology $H^{3}\left(Y, W \mathcal{O}_{Y}\right)$ is the Dieudonné module for the formal group $\Phi_{Y}^{3}$. 


\section{CAlabi-Yau Threefolds}

\section{ACKnowledgements}

The author wishes to thank Stefan Müller-Stach, Klaus Künneman, Uwe Jannsen, and Jean-Marc Fontaine for helpful discussions. He is also grateful to the referee, who suggested several simplifications and pointed out some errors in the first version.

\section{REFERENCES}

Art74 M. Artin, Supersingular K3 surfaces, Ann. Sci. École Norm. Sup. (4) 7 (1974), 543-567.

AM77 M. Artin and B. Mazur, Formal groups arising from algebraic varieties, Ann. Sci. École Norm. Sup. (4) 10 (1977), 87-131.

Bea83 A. Beauville, Variétés Kähleriennes dont la première classe de Chern est nulle, J. Differential Geom. 18 (1983), 755-782.

Bog78 F. Bogomolov, Hamiltonian Kählerian manifolds, Dokl. Akad. Nauk SSSR 243 (1978), 1101-1104.

BM77 E. Bombieri and D. Mumford, Enriques' classification of surfaces in char. $p$ II, in Complex analysis and algebraic geometry, eds W. Baily and T. Shioda (Cambridge University Press, Cambridge, 1977), 23-42.

Del81 P. Deligne, Relèvement des surfaces K3 en caractéristique nulle, in Surface algébrique, eds J. Giraud, L. Illusie and M. Raynaud, Lecture Notes in Mathematics, vol. 868 (Springer, Berlin, 1981), 58-79.

Eis95 D. Eisenbud, Commutative algebra, Graduate Texts in Mathematics, vol. 150 (Springer, New York, 1995).

FK88 E. Freitag and R. Kiehl, Étale cohomology and the Weil conjecture, Ergeb. Math. Grenzgeb. (3), vol. 13 (Springer, Berlin, 1988).

Gro68 A. Grothendieck, Le groupe de Brauer II, in Dix exposés sur la cohomologie des schémas eds J. Giraud et al. (North-Holland, Amsterdam, 1968), 88-189.

Gro70 A. Grothendieck, Schemas en groupes I, Lecture Notes in Mathematics, vol. 151 (Springer, Berlin, 1970).

Gro71a A. Grothendieck, Revêtements étales et groupe fondamental, Lecture Notes in Mathematics, vol. 224 (Springer, Berlin, 1971).

Gro71b A. Grothendieck, Théorie des intersections et théorème de Riemann-Roch, Lecture Notes in Mathematics, vol. 225 (Springer, Berlin, 1971).

Har94 R. Hartshorne, Generalised divisors on Gorenstein schemes, K-Theory 8 (1994), 287-339.

Hir99 M. Hirokado, A non-liftable Calabi-Yau threefold in characteristic 3, Tohoku Math. J. (2) 51 (1999), 479-487.

Ill71 L. Illusie, Complexe cotangent et déformations I, Lecture Notes in Mathematics, vol. 239 (Springer, Berlin, 1971).

Ill79 L Illusie, Complexe de de Rham-Witt et cohomologie cristalline, Ann. Sci. École Norm. Sup. (4) 12 (1979), 501-661.

Kat78 T. Katsura, On Kummer surfaces in characteristic 2, in Proc. Int. Symp. Alg. Geom., ed. M. Nagata (Kinokuniya Book Store, Tokyo, 1978), 525-542.

Kat87 T. Katsura, Generalized Kummer surfaces and their unirationality in characteristic p, J. Fac. Sci. Univ. Tokyo Sect. 34 (1987), 1-41.

KM74 N. Katz and W. Messing, Some consequences of the Riemann hypothesis for varieties over finite fields, Invent. Math. 23 (1974), 73-77.

Kaw92 Y. Kawamata, Unobstructed deformations. A remark on a paper of Z. Ran: 'Deformations of manifolds with torsion or negative canonical bundle', J. Algebraic Geom. 1 (1992), 183-190.

Mor79 L. Moret-Bailly, Polarisations de degré 4 sur les surfaces abéliennes, C. R. Acad. Sci. Paris 289 (1979), A787-A790. 


\section{Calabi-Yau threefolds}

Mor81 L. Moret-Bailly, Familles de courbes et de varietes abeliennes sur $P^{1}$, in Séminaire sur les pinceaux de courbes de genre au moins deux, ed. L. Szpiro, Astérisque 86 (1981), 109-140.

Mum70 D. Mumford, Abelian varieties, Tata Institute of Fundamental Research Studies in Mathematics, vol. 5 (Oxford University Press, Oxford, 1970).

Oor75 F. Oort, Which abelian surfaces are products of elliptic curves? Math. Ann. 214 (1975), 35-47.

Ran92 Z. Ran, Deformations of manifolds with torsion or negative canonical bundle, J. Algebraic Geom. 1 (1992), 279-291.

Sch03 S. Schröer, The $T^{1}$-lifting theorem in positive characteristics, J. Algebraic Geom. 12 (2003), 699-714.

Ser65 J.-P. Serre, Algèbre locale. Multiplicités, Lecture Notes in Mathematics, vol. 11 (Springer, Berlin, $1965)$.

Ser79 J.-P. Serre, Local fields. Graduate Texts in Mathematics, vol. 67 (Springer, Berlin, 1979).

Shi74 T. Shioda, Kummer surfaces in characteristic 2, Proc. Japan Acad. Ser. A Math Sci. 50 (1974), $718-722$.

Shi79 T. Shioda, Supersingular K3 surfaces, in Algebraic geometry, ed. K. Lonsted, Lecture Notes in Mathematics, vol. 732 (Springer, Berlin, 1979), 564-591.

Tia87 G. Tian, Smoothness of the universal deformation space of compact Calabi-Yau manifolds and its Petersson-Weil metric, in Mathematical aspects of string theory, ed. S. Yau, Advanced Series in Mathematics and Physics, vol. 1 (World Scientific, Singapore, 1987), 629-646.

Tod80 A. Todorov, Applications of the Kähler-Einstein-Calabi-Yau metric to moduli of K3 surfaces, Invent. Math. 61 (1980), 251-265.

VK00 G. van der Geer and T. Katsura, On a stratification of the moduli of K3 surfaces, J. Eur. Math. Soc. 2 (2000), 259-290.

Stefan Schröer stefan.schroeer@uni-bayreuth.de

Mathematisches Institut, Universität Bayreuth, 95440 Bayreuth, Germany 